\title{
AVALIAÇÃO DA OCORRÊNCIA SAZONAL DE PERCEVEJOS EM CINCO ESPÉCIES DE MARACUJAZEIRO, UTILIZANDO DOIS MÉTODOS DE AMOSTRAGEM ${ }^{(1)}$
}

\author{
ANTONIO CESAR CAETANO ${ }^{(2)}$; ARLINDO LEAL BOIÇA JÚNIOR ${ }^{(3)}$; \\ CARLOS RUGGIERO ${ }^{(4)}$ \\ RESUMO
}

\begin{abstract}
O presente trabalho teve por objetivo estudar a ocorrência sazonal de percevejos em espécies de maracujazeiro (Passiflora spp.) através de dois métodos de amostragem: número de percevejos por $0,5 \mathrm{~m}^{2}$ e em $1,5 \mathrm{~m}$ linear de espaldeira. $\mathrm{O}$ experimento foi conduzido em pomar de maracujazeiro pertencente à Faculdade de Ciências Agrárias e Veterinárias - UNESP, Jaboticabal, Estado de São Paulo, entre setembro de 1997 e setembro de 1998. Foram utilizadas cinco espécies de maracujazeiro: P. alata, P. edulis f. flavicarpa, $P$. nitida, $P$. giberti e P. laurifolia. Após as avaliações semanais pôde-se concluir que as espécies de percevejo Leptoglossus gonagra, L. zonatus, Holhymenia histrio e Anisoscelis foliacea marginella foram as mais abundantes em maracujazeiro. L. gonagra, H. histrio e A. foliacea marginella apresentaram maiores populações entre o final de dezembro e início de maio e $L$. zonatus ocorreu em maior população entre o final de março e o de julho. $\mathrm{O}$ método de $0,5 \mathrm{~m}^{2}$ foi o mais adequado para avaliação de percevejos. P. laurifolia foi mais atrativo para L. gonagra, P. edulis f. flavicarpa mais atrativo para L. zonatus e $P$. giberti para A. foliacea marginella e H. histrio.

Palavras-chave: Passiflora spp., Coreidae, Leptoglossus, Holhymenia, Anisoscelis.
\end{abstract}

\section{ABSTRACT \\ EVALUATION OF SEASONAL STINKBUG OCCURRENCE IN FIVE PASSION FRUIT SPECIES USING TWO SAMPLING METHODS}

Stinkbug population dynamics has been studying in passion fruit (Passiflora spp.) under field conditions in Jaboticabal, São Paulo State. Two sampling methods have been utilized: a) countings of the number of insects occurring in a $0.5 \mathrm{~m}^{2}$ wooden frame; $b$ ) countings of the number of insects present in 1.5 linear meter, aside of a tutoring system. Five passion fruit species (Passiflora alata, P. edulis $f$. flavicarpa, $P$. nitida, P. giberti and P. laurifolia) have been checked as to insect occurrence throughout a year. Weekly, insect samples have been collected in 5 randomly choosen sites in the orchard, for each evolution method.The most frequent stinkbug species, considering all the passion fruit species, have been the following: Leptoglossus gonagra, L. zonatus, Holhymenia histrio and Anisoscelis foliacea marginella; the highest populations for L. gonagra, H. histrio and A. foliacea marginella have occurred from late December to early May while those of $L$. zonatus revealed the highest numbers from late March to late July. P. laurifolia has been the most attractive species to L. gonagra; P. edulis f. flavicarpa to L. zonatus; $P$. giberti to $A$. foliacea marginella and $H$. histrio.The sampling method using a $0.5 \mathrm{~m}^{2}$ wooden frame has shown to be the most effective as to population dynamics evolutions.

Key words: Passiflora spp., Coreidae, Leptoglossus, Holhymenia, Anisoscelis.

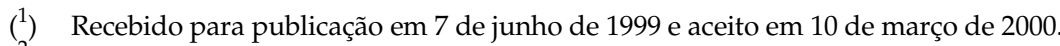

( ${ }^{2}$ Acadêmico do Curso de Pós-Graduação em Produção Vegetal da Faculdade de Ciências Agrárias e Veterinárias da Universidade Estadual Paulista (FCAV/UNESP), Via de Acesso Prof. Paulo D. Castellane, km 5, 14870-000 Jaboticabal (SP).

$\left({ }^{3}\right)$ Departamento de Fitossanidade, FCAV/UNESP, Jaboticabal (SP). E-mail:aboicajr@fcav.unesp.br

$\left({ }^{4}\right)$ Departamento de Horticultura, FCAV/UNESP, Jaboticabal (SP). 


\section{INTRODUÇÃO}

O maracujazeiro (Passiflora spp.) é originário de regiões tropicais, provavelmente da América Latina, e o seu cultivo no Brasil tem sido bem sucedido em regiões similares inclusive em algumas subtropicais (NASCIMENTO, 1997).

Os percevejos são considerados as principais pragas do maracujazeiro adulto em função da sua agilidade, de seu fácil deslocamento, da existência de plantas hospedeiras alternativas, além dos danos que os mesmos causam ao sugarem os botões florais e frutos novos, provocando sua queda (MARICONI, 1952; RUGGIERO et al., 1996; BOIÇA JúNIOR, 1998).

Segundo TeIXeIra (1994), Ruggiero et al. (1996) e BOIÇA JÚNIOR (1998), as principais espécies de percevejos que danificam o maracujazeiro são: Diactor bilineatus (Fabr., 1803), Holhymenia clavigera (Herbst., 1784), Leptoglossus gonagra (Fabr., 1775) e Leptoglossus stigma (Herbst., 1784), os quais pertencem à família Coreidae. Encontram-se ainda com menor freqüência outras espécies citadas na literatura, tais como: $H$. histrio (Fabr., 1803), Anisoscelis foliaceus (Fabr., 1803) (Hemiptera: Coreidae) e Gargaphia lunulata (Mayr, 1865) (Hemiptera: Tingidae) (Rossetto et al., 1974; BorçAJúNIOR, 1998).

No estudo de flutuações populacionais de insetos inúmeros aspectos devem ser observados destacando-se entre eles o método de amostragem. Cassino e DALсомо (1976) basearam seu método em contagens semanais de insetos em áreas de $1 \mathrm{~m}^{2}$, escolhidas ao acaso, e constataram a ocorrência de $D$. bilineatus e Anisoscelis sp. LinHAREs et al. (1983), utilizando o mesmo método de CASSINO E DALCOMO (1976), observaram os percevejos D. bilineatus, $H$. clavigera, $A$. foliaceus e $G$. lunulata.

NoronHA et al. (1998) realizaram um levantamento das populações de artrópodes em maracujazeiro amarelo, P. edulis f. flavicarpa, através de avaliações quinzenais feitas visualmente em duas plantas por linha de plantio, escolhidas ao acaso. Dentre os percevejos observados foram constatados os das espécies D. bilineatus e H. histrio.

No manejo das pragas e doenças do maracujazeiro é importante considerar os insetos polinizadores, pois, em lavouras onde não existem mamangavas, a polinização tem que ser feita manualmente, o que aumenta os custos de produção. Assim, em função dos danos causados por percevejos e da necessidade de se desenvolverem métodos de controle mais eficientes para esse tipo de praga, estudos de ocorrência sazonal são de fundamental importância a fim de permitir maior eficácia na aplicação de defensivos, bem como tornar mais eficiente o manejo integrado.
Sendo assim, o objetivo do presente trabalho foi estudar a ocorrência sazonal de percevejos em cinco espécies de maracujazeiro em Jaboticabal, Estado de São Paulo, através de dois métodos de amostragem.

\section{MATERIAL E MÉTODOS}

O estudo da ocorrência sazonal foi realizado no pomar experimental de maracujá que constitui o Banco Ativo de Germoplasma do Departamento de Fitotecnia da Faculdade de Ciências Agrárias e Veterinárias/UNESP, em Jaboticabal, cujo sistema de condução e sustentação da planta é do tipo espaldeira vertical. O pomar está localizado, geograficamente, a $22^{\circ} 15^{\prime} 22^{\prime \prime} \mathrm{S}$ e $48^{\circ} 18^{\prime} 58^{\prime \prime} \mathrm{W}$ em altitude média de $595 \mathrm{~m}$. Foram utilizadas as seguintes espécies de maracujazeiro para o estudo: Passiflora alata, P. edulis f. flavicarpa, P. nitida, P. giberti e P. laurifolia. As espécies foram escolhidas por serem as mais abundantes do pomar (mais de 15 plantas para cada espécie), além de apresentarem interesse econômico e/ou para fins de melhoramento genético.

A ocorrência sazonal de percevejos foi avaliada no período de 24/09/97 a 22/09/98, completando 52 avaliações semanais para as espécies de maracujazeiro acima citadas, excetuando-se P. laurifolia, na qual as avaliações ficaram restritas ao período de $14 / 01 / 98$ a 22/09/98. Tal fato ocorreu porque na época de planejamento e início das avaliações as plantas desta espécie já estavam com mais de um ano de idade, sem ainda apresentarem florescimento e frutificação. Todas as plantas apresentavam mais de um ano de idade no início das avaliações.

Os elementos meteorológicos utilizados neste trabalho foram extraídos de um conjunto de dados pertencentes ao acervo da área de Agrometeorologia do Departamento de Ciências Exatas da FCAV-UNESP, obtendo-se as médias mensais dos dados de temperatura, precipitação e umidade relativa.

Os insetos das diferentes espécies observadas no campo foram coletados, montados em alfinetes entomológicos e enviados para identificação.

\subsection{Metodologia de amostragem}

As amostragens foram feitas semanalmente através de dois métodos: pelo uso de um quadro de madeira $\left(0,5 \mathrm{~m}^{2}\right)$ e por meio da contagem do número de percevejos em 1,5 m linear, em uma lateral da espaldeira. Para cada uma das espécies de maracujazeiro foram escolhidos cinco pontos ao acaso, no pomar, para cada método de amostragem. Preferiu-se realizar as amostragens no período da tarde, quando as flores de todas as espécies de maracujazeiro estudadas encontravam-se abertas. 
Confeccionou-se um quadro retangular de madeira com $0,5 \mathrm{~m}^{2}$ de área interna livre $(0,50 \mathrm{~m}$ de altura por 1,00 m de comprimento). O quadro foi pendurado na lateral da espaldeira com o auxílio de um gancho de metal, e contou-se o número de percevejos dentro dessa área, anotando-se também a presença de botões, flores e frutos.

Com o auxílio de uma trena foi medido no chão, paralelamente à espaldeira, um metro e meio linear $(1,5 \mathrm{~m})$. A partir das extremidades desse $1,5 \mathrm{~m}$ foram traçadas duas linhas imaginárias verticais que determinavam a área a ser amostrada, na espaldeira, onde foram avaliados os mesmos parâmetros do item anterior.

A partir da $19^{a}$ semana de amostragem foram anotados os locais onde se encontravam os percevejos, a fim de se verificar a preferência dos insetos por algum tipo de estrutura vegetal.

\subsection{Análise estatística}

Os dados obtidos quanto ao número de insetos de cada espécie de percevejo, de acordo com o método utilizado, foram transformados em $\sqrt{\mathrm{x}+0,5}$ e submetidos à análise de variância comparando-se as médias pelo teste de Tukey a 5\%. Por sua vez, os dados referentes ao número total de insetos de cada espécie, bem como o número total de percevejos encontrados (utilizando-se cada um dos métodos), foram comparados pelo teste de Homocedasticidade, de Bartlett.

\section{RESULTADOS E DISCUSSÃO}

\subsection{Métodos de amostragem}

Utilizando-se o método do quadro de madeira foi observada baixa infestação de L. gonagra em P. giberti,
P. nitida, P. edulis f. flavicarpa e $P$. alata, ao passo que um número maior dos mesmos insetos foi observado em P. laurifolia, a qual diferiu significativamente das espécies anteriores (Quadro 1). Quando feita a avaliação em $1,5 \mathrm{~m}$ linear de espaldeira, também os menores números médios de L. gonagra foram encontrados em $P$. edulis f. flavicarpa, P. nitida, P. giberti e P. alata, que diferiram significativamente de $P$. laurifolia, evidenciando certa preferência do inseto por esta última espécie de maracujazeiro. Ao longo do ano o maior número de indivíduos nas cinco espécies de maracujazeiro foi observado entre as semanas $18(20 / 01)$ e 29 (08/04), com o maior pico na $24^{\text {a }}$ semana $(05 / 03)$ para o quadro de madeira (figura 1) e na $27^{\text {a }}$ semana $(26 / 03)$ para $1,5 \mathrm{~m}$ linear de espaldeira (Figura 2). $\mathrm{O}$ período coincidiu com a época de florescimento e frutificação dessas espécies de maracujazeiro (SousA e MeletTI, 1997), o que torna tais percevejos altamente prejudiciais à cultura. Coincidiu também com os meses de maior precipitação média, acima de $100 \mathrm{~mm}$, e maior temperatura média, próxima a $25^{\circ} \mathrm{C}$. A umidade relativa média não ficou abaixo de $50 \%$ em nenhum dos doze meses estudados. Durante o período avaliado (como era de se esperar), o número de insetos observados utilizando-se o método de 1,5 m linear foi superior ao do quadro, visto que envolve uma área muito maior (Quadro 1).

Até a $17^{a}$ semana as avaliações foram feitas apenas nas quatro espécies iniciais e, a partir daí, também em P. laurifolia.

Leptoglossus zonatus (DALLAS, 1852) (Hemiptera: Coreidae), espécie não encontrada na literatura como praga de maracujá, ocorreu com índice populacional superior ao das outras espécies, ficando abaixo somente de L. gonagra (figuras 1 e 2). Para ambos os

Quadro 1. Número médio de percevejos em cinco espécies de maracujazeiro amostrados de 24 de setembro de 1997 a 22 de setembro de 1998 em Jaboticabal (SP), utilizando-se dois métodos de amostragem

\begin{tabular}{lcccccccc}
\hline & \multicolumn{4}{c}{ Quadro de madeira } \\
\cline { 2 - 8 } Espécies & \multicolumn{4}{c}{ Percevejos $^{(1)}$} & \multicolumn{3}{c}{1,5 m linear de espaldeira } \\
\cline { 2 - 8 } & L. gonagra & H. histrio & A.f. marginella & L. zonatus & L. gonagra & H. histrio & A.f. marginella & L. zonatus \\
\hline P. laurifolia & $13,4 \mathrm{a}^{(2)}$ & $0,2 \mathrm{~b}$ & $0,0 \mathrm{~b}$ & $1,2 \mathrm{~b}$ & $23,4 \mathrm{a}$ & $1,8 \mathrm{a}$ & $0,0 \mathrm{~b}$ & $1,8 \mathrm{~b}$ \\
P. edulis & $1,6 \mathrm{~b}$ & $0,0 \mathrm{~b}$ & $0,0 \mathrm{~b}$ & $4,4 \mathrm{a}$ & $1,8 \mathrm{~b}$ & $0,2 \mathrm{~b}$ & $0,2 \mathrm{~b}$ & $8,8 \mathrm{a}$ \\
P. giberti & $1,2 \mathrm{~b}$ & $1,4 \mathrm{a}$ & $3,2 \mathrm{a}$ & $0,2 \mathrm{~b}$ & $4,6 \mathrm{~b}$ & $3,6 \mathrm{a}$ & $7,8 \mathrm{a}$ & $0,4 \mathrm{~b}$ \\
P. alata & $2,8 \mathrm{~b}$ & $0,4 \mathrm{ab}$ & $0,4 \mathrm{~b}$ & $0,4 \mathrm{~b}$ & $4,6 \mathrm{~b}$ & $1,8 \mathrm{a}$ & $1,2 \mathrm{~b}$ & $0,4 \mathrm{~b}$ \\
P. nitida & $1,2 \mathrm{~b}$ & $0,0 \mathrm{~b}$ & $0,0 \mathrm{~b}$ & $0,0 \mathrm{~b}$ & $3,0 \mathrm{~b}$ & $0,2 \mathrm{~b}$ & $0,0 \mathrm{~b}$ & $0,0 \mathrm{~b}$ \\
F & $8,72^{* *}$ & $5,21^{* *}$ & $31,01^{* *}$ & $11,64^{* *}$ & $17,45^{* *}$ & $12,93^{* *}$ & $25,99^{* *}$ & $13,69^{* *}$ \\
C.V.(\%) & 42,43 & 28,55 & 20,89 & 32,85 & 29,26 & 23,92 & 31,58 & 39,85 \\
\hline
\end{tabular}

${ }^{(1)}$ Dados reais. Os dados foram transformados em $\sqrt{x+0,5}$, para fins de análise estatística.

** significativo a $1 \%$ pelo teste $\mathrm{F}$.

${ }^{(2)}$ Médias seguidas de mesma letra, dentro da mesma coluna, não diferem estatisticamente, entre si, pelo teste de Tukey a 5\%. 


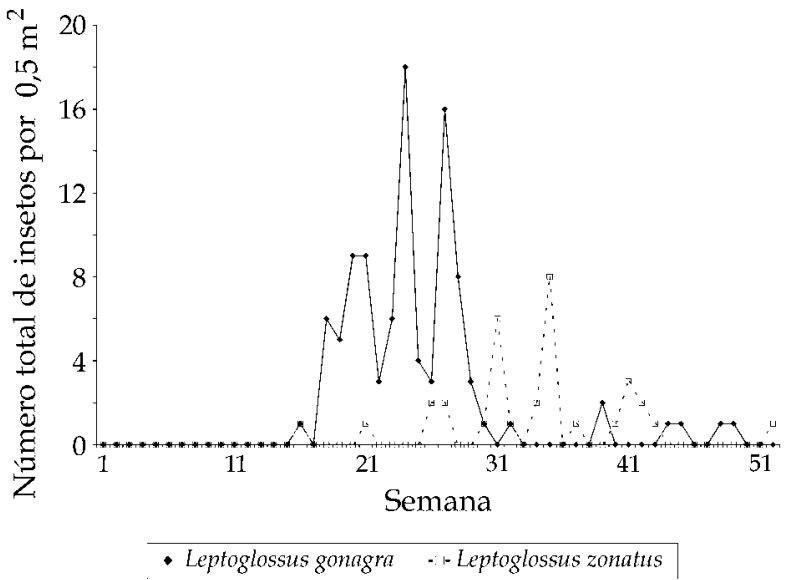

Figura 1. Ocorrência sazonal de Leptoglossus gonagra e Leptoglossus zonatus em cinco espécies de maracujazeiro, no período de 24 de setembro de 1997 a 22 de setembro de 1998 em Jaboticabal, utilizando-se o método de amostragem do quadro de madeira.

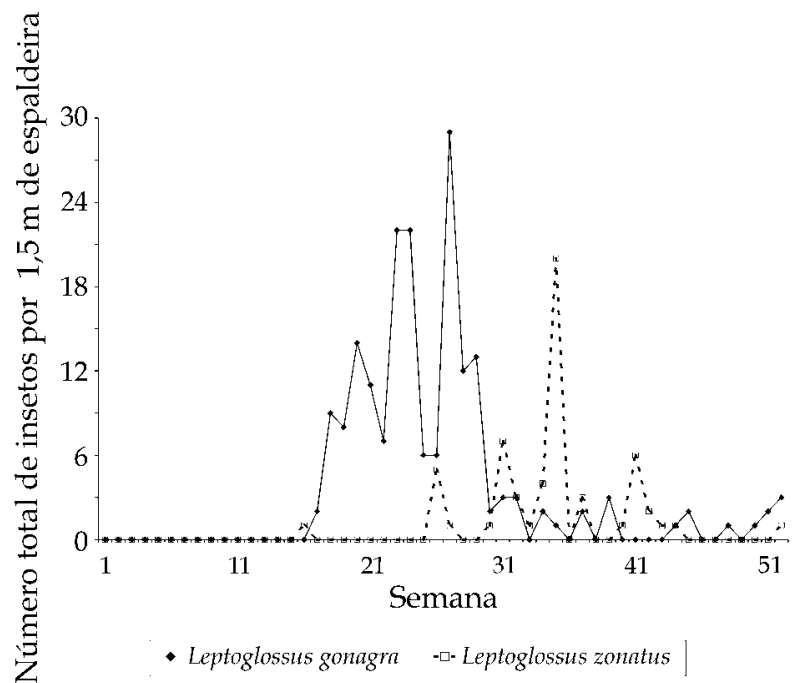

Figura 2. Ocorrência sazonal de Leptoglossus gonagra e Leptoglossus zonatus em cinco espécies de maracujazeiro, no período de 24 de setembro de 1997 a 22 de setembro de 1998 em Jaboticabal, utilizando-se o método de amostragem de 1,5 m linear de espaldeira.

métodos constata-se que a população aumentou a partir da $26^{\text {a }}$ semana (20/03), com o maior pico populacional na $35^{\mathrm{a}}$ semana $(21 / 05)$. Houve pequena diferença entre os métodos de amostragem, quanto ao período em que a população diminuiu: para o método de $1,5 \mathrm{~m}$ linear a redução ocorreu a partir da $41^{\mathrm{a}}$ semana (03/07) e, para o quadro de madeira, a partir da $43^{\text {a }}$ semana (15/07). A ocorrência dessa espécie talvez possa ser explicada pela existência de uma área plantada com milho próxima ao pomar. Quando as plantas de milho começaram a secar, os insetos, vistos em abundância sobre tal cultura, provavelmente mi- graram para as plantas de maracujá. L. Zonatus não foi observado em plantas de P. nitida, sendo encontrado poucas vezes sobre $P$. giberti, $P$. alata e $P$. laurifolia e, em maior população, em $P$. edulis $\mathrm{f}$. flavicarpa (Quadro 1). Nesse período de maior ocorrência a precipitação média foi de cerca de $55 \mathrm{~mm}$ nos meses de abril e maio e sem chuvas durante junho e julho, com a temperatura média inferior a $20^{\circ} \mathrm{C}$.

A época de maior ocorrência de $H$. histrio nas várias espécies de maracujá, utilizando-se o método do quadro de madeira, deu-se entre 30/12 (semana 15) e 21/05 (semana 35) (Figura 3), não tendo sido encontrado qualquer exemplar de Holhymenia sobre P. edulis f. flavicarpa e P. nitida (Quadro 1). Por outro lado, através do método de 1,5 m linear (Figura 4), a época de maior ocorrência foi entre $30 / 12$ ( $15^{a}$ semana) e 08/04 (29a semana), tendo este método uma diferença de seis semanas em relação ao anterior. Apesar de o número de insetos ter sido baixo durante todo o ano percebe-se que houve maior número deles em $P$. giberti, a qual diferiu significativamente de $P$. laurifolia, $P$. edulis $f$. flavicarpa e $P$. nitida, enquanto $P$. alata apresentou tendência intermediária. Pelos dados obtidos por BALDIN (1998) - que avaliou a atratividade de espécies de maracujazeiro para este percevejo, em laboratório - seria esperado um número de insetos igual, ou menor, em $P$. giberti. É provável que isso não tenha ocorrido devido à atuação de vários outros estímulos sobre o inseto (em condições de campo), os quais são eliminados (em laboratório) por condições ambientais artificiais ou por estresse dos insetos. Esse percevejo foi observado sobre plantas de maracujá amarelo somente quando se utilizou o método de $1,5 \mathrm{~m}$ linear. Apesar de estar de acordo com os relatos de NoronHa et al. (1998), o baixo número de indivíduos encontrados sobre essa planta evidencia uma preferência maior do inseto por outras espécies de maracujazeiro, neste caso, $P$. giberti.

Constatou-se que a ocorrência de $A$. foliacea marginella foi esporádica em $P$. alata e $P$. edulis f. flavicarpa e teve maior número de exemplares sobre $P$. giberti (Quadro 1), enquanto nas demais espécies não se observou qualquer inseto. Pela amostragem de $0,5 \mathrm{~m}^{2}$, nota-se que a época de maior ocorrência desse percevejo assemelhou-se às anteriores (Figura 3 ), ou seja, iniciou-se na $16^{\mathrm{a}}$ semana $(06 / 01)$ e estendeu-se até a $40^{\mathrm{a}}$ semana $(26 / 06)$, com o maior pico na $20^{\mathrm{a}}$ semana (04/02). Quando utilizado 1,5 m linear (Figura 4), verificou-se que o inseto ocorreu em maior intensidade entre a $15^{\mathrm{a}}(30 / 12)$ e a $29^{\mathrm{a}}(08 / 04)$ semana, dez a menos que no caso anterior. Tais resultados estão de acordo com os relatos de CASSINO e DALCOMO (1976) e LiNHARES et al. (1983), que observaram o pico populacional de percevejos desse mesmo gênero 
durante a época de floração do maracujazeiro, com sua ocorrência estendendo-se até o fim ou um pouco após a floração.

Deve-se destacar, ainda, que outras espécies de percevejo, tais como Phthia picta (Drury, 1770) e Acanthocephala sp. (Hemiptera: Coreidae), foram observadas ao longo do ano em plantas de maracujazeiro, ainda que esporádicas e em baixas populações.

Além disso, vale ressaltar que não foi constatada neste trabalho a presença de $D$. bilineatus, a espécie de percevejo mais citada na literatura brasileira especializada como um inseto de grande importância para a cultura. Os percevejos coletados durante as avaliações, com alguma semelhança com $D$. bilineatus, foram todos identificados como insetos do gênero Anisoscelis, o que deixa dúvida sobre outros relatos.

Comparando-se, de modo geral, os dois métodos de amostragem utilizados, o método de 1,5 m linear ao longo da espaldeira proporcionou a observação de maior número de insetos durante o período avaliado, em comparação ao do quadro de madeira. Todavia, pela análise estatística pode-se observar que a variância proporcionada pelo quadro foi menor em relação ao outro método, havendo diferença significativa en-

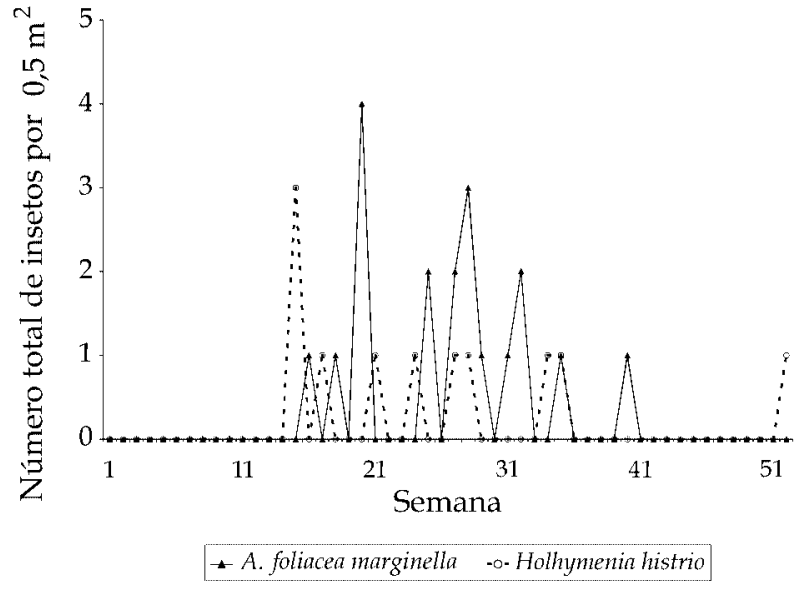

Figura 3. Ocorrência sazonal de Anisoscelis foliacea marginella e Holhymenia histrio em cinco espécies de maracujazeiro no período de 24 de setembro de 1997 a 22 de setembro de 1998 em Jaboticabal, utilizando-se o método de amostragem do quadro de madeira.

tre ambos para H. histrio (Quadro 2). Desse modo, o método do quadro de madeira foi o que apresentou maior homogeneidade ao longo das avaliações. Além disso, seu uso envolve uma área menor a ser avaliada, facilitando e agilizando a amostragem. Sendo assim, esse método pode ser considerado mais adequado em

Quadro 2. Comparação entre a variabilidade em cada método de amostragem do número médio de insetos de cada espécie de percevejo e para percevejos em geral, em maracujazeiro, pelo teste de Bartlett durante o período de setembro de 1997 a setembro de 1998

\begin{tabular}{|c|c|c|c|c|}
\hline \multirow[t]{2}{*}{ Espécie } & & \multicolumn{3}{|c|}{ Método } \\
\hline & & $1,5 \mathrm{~m}$ linear & vs. & Quadro \\
\hline \multirow{3}{*}{ L. gonagra } & Média & $37,40 \mathrm{a}$ & & $20,20 a$ \\
\hline & Variância & 88,30 & & 64,70 \\
\hline & $\mathrm{E}$ & & $0,86^{\mathrm{ns}}$ & \\
\hline \multirow{3}{*}{ L. zonatus } & Média & $11,40 \mathrm{a}$ & & $6,20 \mathrm{a}$ \\
\hline & Variância & 36,30 & & 19,20 \\
\hline & E & & $0,35^{\mathrm{ns}}$ & \\
\hline \multirow{3}{*}{ A. f. marginella } & Média & $9,20 \mathrm{a}$ & & $4,00 \mathrm{a}$ \\
\hline & Variância & 13,70 & & 2,00 \\
\hline & $\mathrm{E}$ & & $2,88^{\mathrm{ns}}$ & \\
\hline \multirow{3}{*}{ H. histrio } & Média & $7,60 \mathrm{a}$ & & $2,00 \mathrm{~b}$ \\
\hline & Variância & 12,80 & & 0,50 \\
\hline & $\mathrm{E}$ & & $6,87^{*}$ & \\
\hline \multirow{3}{*}{ Total de percevejos } & Média & $65,60 a$ & & $32,00 \mathrm{a}$ \\
\hline & Variância & 188,30 & & 76,00 \\
\hline & $\mathrm{E}$ & & $0,71^{\mathrm{ns}}$ & \\
\hline
\end{tabular}

Estatística do teste de homocedasticidade de Bartlett.

* significativo a 5\%; ns - não significativo pelo teste de Bartlett.

Médias seguidas de mesma letra, dentro das linhas, não diferem, entre si, pelo teste do $X^{2}$ a $5 \%$. 


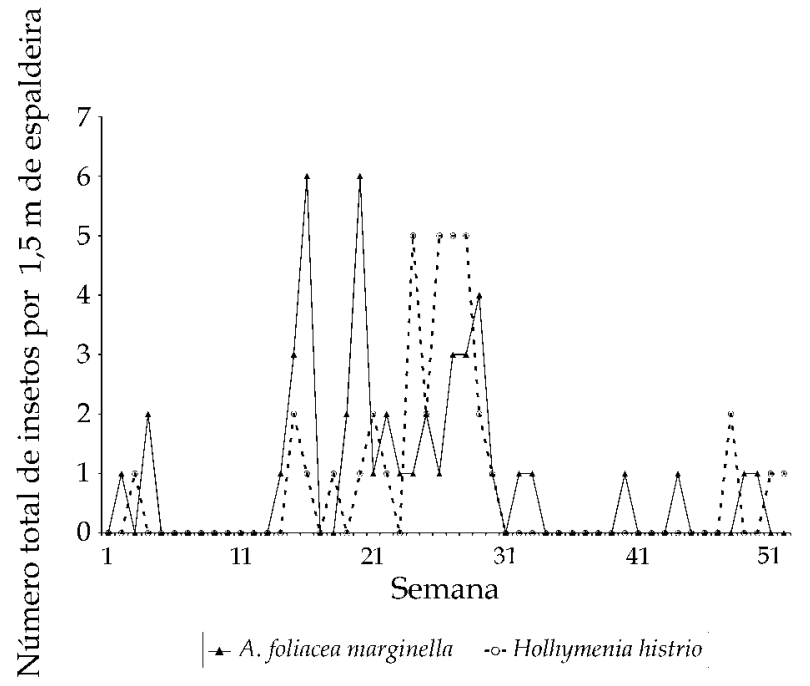

Figura 4. Ocorrência sazonal de Anisoscelis foliacea marginella e Holhymenia histrio em cinco espécies de maracujazeiro no período de 24 de setembro de 1997 a 22 de setembro de 1998 em Jaboticabal, utilizando-se o método de amostragem de 1,5 m linear de espaldeira.

relação ao de 1,5 m linear para amostragem de percevejos.

\subsection{Distribuição de insetos na planta}

De um total de 320 insetos observados a partir da $19^{a}$ semana de avaliação, nas cinco espécies de maracujá, notou-se a incidência de insetos em cada parte da planta: foram observados 5 percevejos sobre flores, 24 sobre as hastes dos ramos, 29 em botões florais, 52 nas folhas e 210 insetos sobre frutos. Assim, aproximadamente $67 \%$ dos insetos amostrados encontravam-se sobre frutos, o que comprova sua preferência por este tipo de estrutura vegetal, concordando com os relatos de MARiconi (1952). Dos 210 percevejos encontrados sobre frutos, 109 foram observados sobre P. laurifolia, e, desse total, 98 eram L. gonagra, o que indica uma preferência desse percevejo por frutos dessa espécie.

\section{CONCLUSÕES}

1. L. gonagra, L. zonatus, A. foliacea marginella e $H$. histrio são as espécies de percevejos que ocorrem com maior freqüência em plantas de maracujazeiro em Jaboticabal, sendo L. gonagra a mais abundante, seguida de L. zonatus;

2. L. gonagra, H. histrio e A. foliacea marginella apresentam maiores populações entre o final de dezembro e início de maio, enquanto L. zonatus apresenta maior população entre o final de março e o de julho;

3. o método de amostragem do quadro de madeira é adequado para se avaliar a infestação de percevejos em maracujazeiro;
4. P. laurifolia foi mais atrativo a L. gonagra, enquanto $P$. edulis f. flavicarpa foi mais atrativo para $L$. zonatus e $P$. giberti foi mais atrativo para A. foliacea marginella e H. histrio.

\section{AGRADECIMENTOS}

À CAPES pela bolsa de Mestrado concedida ao primeiro autor; ao Prof. Dr. João Carlos de Oliveira pela cessão do pomar experimental e ao Prof. Dr. José Carlos Barbosa pelo auxílio nas análises estatísticas, ambos da FCAV-UNESP; à Prof ${ }^{\mathrm{a}}$. Dra. Jocélia Grazia e a seu orientado, Dr. José Antonio Marin Fernandes, da Universidade Federal do Rio Grande do Sul, pela identificação das espécies de inseto coletadas.

\section{REFERÊNCIAS BIBLIOGRÁFICAS}

BALDIN, E.L.L. Tipos de resistência de genótipos de maracujazeiro a Holhymenia histrio (Fabr., 1803) (Hemiptera: Coreidae). Jaboticabal, 1998. 111p . Dissertação (Mestrado em Entomologia Agrícola) - Faculdade de Ciências Agrárias e Veterinárias, Universidade Estadual Paulista.

BOIÇA JÚNIOR, A.L. Pragas da cultura do maracujazeiro. In: RUGGIERO, C. (Coord.) SIMPÓSIO BRASILEIRO SOBRE A CULTURA DO MARACUJÁ, 5., Jaboticabal, 1998. Anais. Jaboticabal, FUNEP, 1998. p.174-203.

CASSINO, C.R.; DALCOMO, L. Flutuação das principais pragas do maracujá no Estado do Rio de Janeiro (Nota Prévia). In: CONGRESSO BRASILEIRO DE ENTOMOLOGIA, 3., Maceió, 1976. Resumos... Jaboticabal, Sociedade Entomológica do Brasil, 1976. p.68-69.

LINHARES, R.P.; MAGNO, P.R.; CASSIANO, P.C.R. Insetos associados ao maracujazeiro, no campus da UFRRJ, Itaguaí-RJ. In: CONGRESSO BRASILEIRO DE ENTOMOLOGIA, 8., Brasília, 1983. Resumos... Brasília, Sociedade Entomológica do Brasil, 1983. p.67.

MARICONI, F.A.M. Contribuição para o conhecimento do Diactor bilineatus (Fabricius, 1803) (Hemiptera Coreidae), praga do maracujazeiro (Passiflora spp.). Arquivos do Instituto Biológico, São Paulo, v.21, p.2144, 1952.

NASCIMENTO, W.A. do. O cultivo do maracujá. Goiânia: EMATER, 1997. 57p. (Boletim Técnico, 1)

NORONHA, A.C.S.; FRANCELLI, M.; JESUS, F.S.; SANTOS, P.A.; MONTE, A.L. Levantamento de artrópodes em plantio de maracujá amarelo no município de Catu, BA. In: RUGGIERO, C. (Coord.) SIMPÓSIO BRASILEIRO SOBRE A CULTURA DO MARACUJÁ, 5., Jaboticabal, 1998. Anais... Jaboticabal, FUNEP, 1998. p.358-359.

ROSSETTO, C.J.; Cavalcante, R.D.; GRISI-JÚNIOR, G.; CARVALHO, A.M. Insetos do maracujazeiro, 
Passiflora spp. In: SIMPÓSIO SOBRE A CULTURA DO MARACUJÁ, 1., Campinas, 1974. Anais... Campinas. CATI, 1974. p.1-19.

RUGGIERO, C.; SÃO JOSÉ, A.R.; VOLPE, C.A.; OLIVEIRA, J.C.; DURIGAN, J.F.; BAUMGARTNER, J.R.; SILVA, J.R.; NAKAMURA, K.; FERREIRA, M.E.; KAVATI, R.; PEREIRA, V.P. Maracujá para exportação: aspectos técnicos da produção. Brasília: Empresa Brasileira de Pesquisa Agropecuária, 1996. 64p. (FRUPEX, 19)

SOUSA, J.S.I. de; MELETTI, L.M.M. Maracujá: espécies, variedades, cultivo. Piracicaba: FEALQ, 1997. p.15126.

TEIXEIRA, C.G. Cultura. In: ITAL. Maracujá: cultura, matéria-prima, processamento e aspectos econômicos. Campinas, 1994. p.1-142. (Série Frutas Tropicais, 9) 